\section{From the authors:}

We would like to thank P.J.P. Poels, D.P.A. Schellekens and T.R. Schermer for the interest shown in our recent paper [1] and for their comments. It is clear that we all agree that primary-care spirometry is important, albeit currently underused. However, we are unable to concur with one of the basic assumptions of the correspondents, namely that spirometry will automatically lead to improvements in management. Despite demonstrating positive effects on pharmacological and nonpharmacological management, arguably the most striking data from our study relate to undertreatment. Even after spirometric confirmation of chronic obstructive pulmonary disease (COPD), $63 \%$ of subjects were not prescribed an inhaled anticholinergic, 75\% were not prescribed an inhaled long-acting $\beta$-agonist and $89 \%$ of subjects had not completed pulmonary rehabilitation. While not all of these subjects were necessarily overtly symptomatic, $>20 \%$ of COPD patients with a forced expiratory volume in one second $(\mathrm{FEV} 1)<1 \mathrm{~L}$, and $>50 \%$ of subjects with an FEV1 $<60 \%$ predicted, were prescribed neither an anticholinergic nor a long-acting $\beta$-agonist. It is difficult to believe that these subjects were not symptomatic and would not have benefited from the treatment or referral for exercise therapy. The use of specific payments for spirometry testing in primary care is likely to increase the number of tests performed dramatically, albeit at a large financial cost. However, translating such diagnostic information into improved treatment for patients is a huge challenge, more dependent on education and use of guidelines. In our view, studies that addressed this problem would be more valuable than one that establishes the positive and negative predictive value of spirometry testing.
One area in which we agree with the correspondents is the importance of aiding the interpretation of spirometry tests in primary care and of ensuring that the tests performed are accurate and of high quality. This is why we discussed in detail that our model of service was an alternative to testing within primary-care groups, where at times individuals using spirometry have had limited training in accurately performing the test or interpreting the results. In our service model, we produce accurate results and we interpret them for the referrer, and certainly there would also be scope to add general management recommendations. Whether this produces superior outcomes to within-practice testing would, in our view, be another study worth performing.

\section{P.P. Walker* and L. Davies ${ }^{\#}$}

*Division of Infection and Immunity, School of Clinical Sciences, University of Liverpool, and \#Aintree Chest Centre, University Hospital Aintree, Liverpool, UK.

\section{STATEMENT OF INTEREST}

None declared.

\section{REFERENCES}

1 Walker PP, Mitchell P, Diamantea F, Warburton CJ, Davies L. Effect of primary-care spirometry on the diagnosis and management of COPD. Eur Respir J 2006; 28: 945-952.

DOI: $10.1183 / 09031936.00163706$

\title{
Evidence-based medicines for children: ethical aspects
}

\section{To the Editors:}

In an editorial in the December issue of the European Respiratory Journal, BUSH [1] describes and welcomes newly proposed legislation from the European Commission requiring testing of all new medicine in children. We certainly agree that this new proposal is a step forward in terms of developing evidence-based therapies for children. We also agree that the creation of a new Paediatric Committee is an opportunity for the scientific community to influence the direction of research. This Committee could increase the collaboration between industry, researchers, paediatric societies and clinicians to the benefit of diseased children. By granting companies an extended period of patent and data protection, the new proposal reduces some of the economic barriers that have hampered clinical research in the paediatric population.

However, the cost of paediatric trials is not the only barrier that needs to be addressed. Paediatric research is ripe with ethical dilemmas that need to be discussed as an integral part of the implementation of the new legislation. There is a growing body of literature discussing specific ethical dilemmas in relation to paediatric research, such as the inclusion of healthy children in nontherapeutic research $[2,3]$ and the role of children's assent and dissent in decision making [4, 5]. A recent paper discussed the role of altruistic considerations in the informed consent process of paediatric research [6]. While several studies report that altruistic considerations are among the motives that parents often cite when they explain their decision to enrol their child [7-10], it is still debatable whether or not investigators should include the subject of altruism in discussions with parents about participation. An empirical study of 140 audiotaped paediatric informed consent sessions shows that the enrolment rate of children was not higher among children whose parents were involved in discussions of altruism compared with those parents who were not [6]. This is good news in so far as such discussions ought to be nonoppressive but it leaves one wondering what really motivates parents to let their children participate in research. In our opinion, more empirical studies are needed 